anti-resonant dip at the maximum of polariton absorption: the decrease of efficiency indicates that re-absorption effects at exact resonance prevail over the probability enhancement.

(iv) Resonant increase and cooling of polariton fluorescence have been observed and connected with Resonant Brillouin Scattering. In a backscattering configuration, one observes polariton escaping from the crystal with a wave vector opposite to that of the exciting light. Polaritons scattered by one longitudinal acoustic phonon give rise to the well defined Resonant Brillouin Scattering peaks. All other polaritons, having suffered several successive processes are smoothly distributed and give rise to Resonant Polariton Fluorescence. Thus the Resonant Brillouin Scattering involves a memory of the initial state, whereas the Resonant Polariton Scattering originates from a randomised distribution of more or less thermalized phonons.

The experiments carried out on CdS by Winterling and Koteles have determined the dispersion of the exciton-polariton and have led also to the study of the properties of high frequency acoustc phonons and the interaction between phonons and polaritons.

Below the longitudinal exciton energy $E_{L}$ "allowed" longitudinal acoustic phonon and "forbidden" transverse acoustic phonon scattering processes are observed, whose line shifts fit well with the two-branch model of the polariton dispersion.

Above $E_{L}$ a set of narrow strong lines is observed which cannot be explained by the predicted one phonon process based on the two-branch model.

The observation of a new resonance phenomenum was also reported by $M$. Balkanski, C. Hirlimann and J.F. Morhange: replicas of the first-order phonon spectrum ${ }^{6}$ ). The first-order Raman spectrum of mixed III-V semiconductor compounds contains the characteristic normal modes of the two compounds. At resonance conditions, phonon replicas of this spectrum are observed, only in disordered system; the first-order spectrum is entirely reproduced, shifted by the frequency of the longitudinal optical mode. The scattered intensity of a replica can be even higher than that of the first order spectrum depending on the frequency of the incident beam. Such replicas are absent from the spectra of pure crystals.

Resonance occurs at a photon energy close to the band gap. Replicas have been observed also for higherorder LO phonon combinations. These results are interpreted by a model in which the relevant resonant intermediate state is a localized exciton strongly interacting with the LOphonon. The different replica orders correspond to resonance states formed by differently "dressed" trapped excitons.

It is obvious that the choice presented here is personal and purposely limited to only very few of the many high-level, timely and important contributions presented at this very stimulating scientific meeting.

\section{References}

References are to the Proceedings of the International Conference on Lattice Dynamics (Flammarion Sciences, Paris), 1978.

\title{
New President of EPS, Antonino Zichichi
}

Antonino Zichichi, a founding member of EPS, is a high energy physicist, presently engaged on a systematic study of the proton structure at the CERN machines ISR and SPS. Professor of Advanced Physics at the University of Bologna (Italy) he was the Director of the Postdoctoral School of Physics at that University. Zichichi is well known in the international physics community; the study of lepton pairs produced in hadronic interactions, the search of heavy leptons produced in $\mathrm{e}^{+} \mathrm{e}^{-}$annilations, exemplify his pioneering contributions in two branches of sub-nuclear physics where a decade was to pass before the phenomena were understood. His research activity covers many fields: the high precision tests of Quantum Electrodynamics, the systematic study of the electromagnetic form factors of the proton and the pseudscalar mesons, the first measurements of the $\left(w_{-} \varnothing\right)$ mixing angle, the discovery of the antideuteron and of the $2 \delta$ decay of the $X$ meson, the high precision measurement of the weak coupling constant...

$\mathrm{He}$ is the founder and the Director of the "Ettore Majorana" Centre for Scientific Culture, established in 1962 and now consisting of 70 national and international Schools - the same Cen- tre which has put a considerable number of places at the disposal of EPS and has offered advantageous terms to Divisions wishing to hold conferences there. In June 1977 Zichichi was elected President of the italian Istituto Nazionale di Fisica Nucleare (INFN) which promotes fundamental research in sub-nuclear and

nuclear physics. More recently, at the plenary meeting of the European Committee for Future Accelerators, he was elected chairman of the working committee studying the project for a large electron-positron colliding beam device (LEP) that ECFA considers is the European high energy machine for the 1980s.

\section{LASER DEPARTMENT \\ INSTITUTE OF APPLIED PHYSICS, UNIVERSITY OF BERN}

Applications are invited for the position of :

\section{RESEARCH PROJECT LEADER}

Qualifications: Essential - Experience in Gas Laser Physics with preferably a knowledge of electronic transition lasers and related discharge techniques.

Desirable - Some knowledge of German.

The position carries responsibility for the Gas-Laser group (staff complement : $2 \mathrm{PhD}, 1$ dipl. and $1 \mathrm{PhD}$ candidate) which is part of the Laser Department (staff complement : 25).

The Gas-Laser group is presently working on CW high power lasens in the visible and near UV and on pulsed TEA discharges.

Equipment available for studies is the following :

- TEA discharge system for metal vapours

- Powerful UV sources (coherent and incoherent), pulsed and CW dye lasers

- $300 \mathrm{~kW}$ DC power supply (600 A or $1 \mathrm{kV}$ )

Additional information may be obtained trom, and applications accompanied by curricula vitae sent to: Prof. Dr. Heinz. P. Weber

institute of Applied Physics, University of Bern

Sidlerstrasse 5

CH-3012 Bern

The position will be open from 1 July, 1978. 